\title{
Prediction and Prevention of Preterm Birth: A Prospective, Randomized Intervention Trial
}

\author{
D. Ware Branch, $\mathrm{MD}^{1}$ John M. VanBuren, $\mathrm{PhD}^{2} \quad$ T. Flint Porter, MD ${ }^{1}$ Calla Holmgren, MD ${ }^{1}$ \\ Richard Holubkov, PhD ${ }^{2}$ Kent Page, MStat ${ }^{2}$ Julja Burchard, MS ${ }^{3}$ Garrett K. Lam, MD ${ }^{4}$ \\ M. Sean Esplin, MD ${ }^{1}$
}

${ }^{1}$ Department of Obstetrics and Gynecology, Intermountain Healthcare Maternal-Fetal Medicine and University of Utah Health, Murray, Utah

2 Division of Pediatric Critical Care, Department of Pediatrics, University of Utah Health, Murray, Utah

${ }^{3}$ Sera Prognostics, Inc., Department of Research \& Development, Salt Lake City, Utah

Am J Perinatol

\section{Abstract}

Keywords

- spontaneous preterm birth

- serum proteomics screening

- neonatal length of stay

received

December 22, 2020 accepted after revision June 17, 2021

\author{
Address for correspondence D. Ware Branch, MD, Department of \\ Obstetrics and Gynecology, 30 N 1900 E, Room 2B200 SOM, Salt Lake \\ City, UT 84132 (e-mail: ware.branch@imail.org). \\ ${ }^{4}$ High Risk Pregnancy Center, Las Vegas, Nevada
}

Objective The study aimed to determine if a program of mid-trimester serum proteomics screening of women at low risk for spontaneous preterm birth (sPTB) and the use of a PTB risk-reduction protocol in those whose results indicated an increased risk of SPTB would reduce the likelihood of SPTB and its sequelae.

Study Design Prospective comparison of birth outcomes in singleton pregnancies with midtrimester cervical length $\geq 2.5 \mathrm{~cm}$ and at otherwise low risk for sPTB randomized to undergo or not undergo mid-trimester serum proteomics screening for increased risk of sPTB (NCT 03530332). Screen-positive women were offered a group of interventions aimed at reducing the risk of spontaneous PTB. The primary outcome was the rate of SPTB $<37$ weeks, and secondary outcomes were gestational age at delivery, total length of neonatal stay, and NICU length of stay (LOS). Unscreened and screen-negative women received standard care. The adaptive study design targeted a sample size of 3,000 to 10,000 women to detect a reduction in sPTB from 6.4 to $4.7 \%$. Due to limited resources, the trial was stopped early prior to data unblinding.

Results A total of 1,191 women were randomized. Screened and unscreened women were demographically similar. SPTB $<37$ weeks occurred in $2.7 \%$ of screened women and $3.5 \%$ of controls $(p=0.41)$. In the screened compared with the unscreened group, there were no between-group differences in the gestational age at delivery, total length of neonatal stay, and NICU LOS. However, the NICU LOS among infants admitted for $\mathrm{SPTB}$ was significantly shorter (median $=6.8$ days, interquartile range [IQR]: $1.8-8.0$ vs. 45.5 days, IQR: $34.6-79.0 ; p=0.005)$.

Conclusion Mid-trimester serum proteomics screening of women at low risk for sPTB and the use of a SPTB risk-reduction protocol in screen-positive patients did not significantly reduce the rate of SPTB compared with women not screened, though the trial was underpowered thus limiting the interpretation of negative findings. Infants in the screened group had a significantly shorter NICU LOS, a difference likely due to a reduced number of infants in the screened group that delivered $<35$ weeks.
DOI https://doi.org/ 10.1055/s-0041-1732339. ISSN 0735-1631.

\footnotetext{
(C) 2021. The Author(s).

This is an open access article published by Thieme under the terms of the Creative Commons Attribution-NonDerivative-NonCommercial-License, permitting copying and reproduction so long as the original work is given appropriate credit. Contents may not be used for commercial purposes, or adapted, remixed, transformed or built upon. (https://creativecommons.org/ licenses/by-nc-nd/4.0/) Thieme Medical Publishers, Inc., 333 Seventh Avenue, 18th Floor, New York, NY 10001, USA
} 
Prediction and Prevention of Preterm Birth Branch et al.

\section{Key Points}

- Mid-trimester serum proteomics screening of women at low risk for SPTB and the use of a sPTB risk-reduction protocol in screen-positive patients did not significantly reduce the rate of SPTB, though the trial was underpowered.

- NICU LOS following SPTB was significantly shortened among women who underwent screening and risk-reduction management.

- The use of serum biomarkers may contribute to a practical strategy to reduce sPTB sequelae.

Preterm birth (PTB), defined as delivery prior to 37 weeks of gestation, remains the dominant cause of neonatal morbidity and mortality throughout the world, including up to $50 \%$ of pediatric neurodevelopmental disorders. ${ }^{1}$ Infants born prematurely are at increased risk for a variety of long-term medical complications such as respiratory, gastrointestinal, cardiovascular, and metabolic disorders. $^{2,3}$ Interventions that reduce the effects of PTB would have a profound impact on the medical, financial, and emotional burden for these children, their families, and the health care system. ${ }^{4}$

Clinical identification of women at risk of spontaneous PTB (SPTB) has been based on nonspecific socioeconomic factors, a history of prior preterm birth, or detection of a short cervix. ${ }^{4}$ History of a prior SPTB is the strongest predictor $^{5}$; however, $40 \%$ or more of pregnancies occur in nulliparous women, and only $7.2 \%$ of multiparous women have a history of sPTB. ${ }^{6,7}$ Shortening of the cervix occurs in approximately 1 to $2 \%$ of the general population, but this finding has a modest predictive value for SPTB in a nulliparous or general multiparous population. ${ }^{8}$ Socioeconomic and demographic risk factors for SPTB (African-American race, lower socioeconomic status, and extremes of body mass index $)^{4,9}$ are relatively common in the general obstetric population and have low positive predictive values. ${ }^{10}$ The unfortunate reality is that two-thirds of SPTB occur among women without an identifiable risk factor. ${ }^{6}$

Recognizing the limits of using clinical and demographic risk factors to prevent sPTB, ${ }^{11-14}$ researchers have developed serum proteomics assays that can be used to better risk stratify women at otherwise low risk of sPTB. ${ }^{15}$ Differential expression of two proteins (sex hormone binding globulin and insulin-like growth factor-binding protein 4 accurately stratify women who delivered prematurely from those who did not: the AUC was 0.75 for predicting birth prior to 37 weeks, and the AUC was 0.93 for predicting birth prior to 35 weeks. This proteomic test has been verified and validated $^{15}$ and is commercially available (PreTRM, Sera Prognostics, Inc. Salt Lake City, UT). Recently, this same test was found to identify a higher risk of a composite index of neonatal morbidity that includes both medical complications of PTB and NICU LOS. ${ }^{16,17}$

Risk stratification for SPTB using a serum proteomics approach is intriguing, but whether such an approach will prevent SPTB and reduce associated neonatal morbidity is unknown. We sought to assess the clinical utility of the PreTRM blood test in population screening and to determine whether a predefined preterm birth prevention protocol utilized in screen-positive women would lead to a reduction in SPTB.

\section{Materials and Methods}

\section{Trial Design and Procedures}

Ours was an investigator-initiated, prospective, randomized, and controlled study enrolling women of any parity without current or historical risk factors for SPTB, as detailed in the inclusion and exclusion criteria ( - Table 1 ) and with the midtrimester ultrasound finding of a cervical length $\geq 2.5 \mathrm{~cm}$. One randomized arm was screened with the PreTRM test (Sera Prognostics, Salt Lake City, UT) to stratify women as increased risk of sPTB (screen-positive, risk $\geq 14 \%$ ) versus lower risk of SPTB (screen-negative, risk $<14 \%$ ). The other randomized arm was unscreened (the control arm). A randomization sequence was created through block permutations of two, four, and six, stratified by clinical site. Coordinators implemented the randomization process using an online randomization sequence. Outcomes were compared across the entirety of both arms. Enrollment began in May, 2018 at multiple clinical sites, including clinic-based, community-based, and hospital-based locations. All participants were consented prior to 19 to 20 weeks and had a serum sample obtained between $19^{5 / 7}$ and $20^{6 / 7}$ weeks. They were randomly assigned in a 1:1 ratio to have screening with the PreTRM test (Sera Prognostics, Inc.) or not. Gestational age (GA) at blood draw between $19^{5 / 7}$ and $20^{6 / 7}$ falls within clinically validated blood draw range of the PreTRM test. ${ }^{15}$ The unscreened group received standard obstetric care in their community setting. Screen-negative women and those without usable blood samples also received standard obstetric care in their community setting.

A sPTB risk-reduction protocol was designed to include several interventions (including progesterone supplementation, cervical length surveillance, daily low dose aspirin, and weekly contact for review of symptoms) that have been shown to be effective at preventing PTB in other women at high risk for PTB. The protocol offered to screen-positive women included two visits to a preterm birth prevention clinic (at $<24$ weeks and at 26-30 weeks). At the first visit, all participants were evaluated for clinical signs and symptoms of PTB, and were offered weekly contact, including phone call and/or a digital risk-reduction program for sPTB (CareCentra, New York City, NY) and access to 24-hour support. Participants accessed the app-based platform to assess for concerning symptoms using a standardized survey with preprogrammed pathways that trigger clinical evaluations, 
Table 1 Study inclusion and exclusion criteria

\section{Inclusion criteria}

Participant $\geq 18$ y of age

Uncomplicated singleton intrauterine pregnancy $<21 \mathrm{wk}$ of estimated gestation

No medical contraindications to continuing pregnancy

No previous classical cesarean section

No known uterine anomaly

No history of cervical conization

No plan for cesarean section prior to $37^{0 / 7}$ wk of gestation

No plan for induction of labor prior to $37^{0 / 7}$ wk of gestation

No history of spontaneous preterm delivery

No prior PPROM $<34^{0 / 7}$ wk of gestation

No signs and/or symptoms of preterm labor

Intact membranes

No history of blood transfusion during the current pregnancy

\section{Exclusion criteria}

Participants who have taken or planned to take progesterone beyond $13^{6 / 7} \mathrm{wk}$ of gestation prior to study enrollment

Any other medical conditions that put participant at increased risk of preterm birth in the judgment of the site investigator

Planned cerclage placement for the current pregnancy

Previously identified short cervix $(<2.5 \mathrm{~cm}$ by transvaginal ultrasound) prior to enrollment

Known major structural fetal anomalies that may shorten pregnancy (e.g., anencephaly, holoprosencephaly, schizencephaly, gastroschisis, omphalocele, and congenital diaphragmatic hernia)

Known fetal genetic anomalies incompatible with life (e.g., trisomy 13 or trisomy 18)

Known elevated bilirubin levels (hyperbilirubinemia)

The participant has taken or planned to take any of the following medications after the first day of the last menstrual period: enoxaparin, heparin, heparin sodium, low molecular weight heparin, and low dose aspirin

A history of allergic reaction to aspirin or 17-OHPC injections

Abbreviations: PPROM, preterm premature rupture of the membranes; 17-OHPC, 17-alpha hydroxyprogesterone.

persuade compliance with treatment plans (i.e., medication use, visits, etc.), and allow for personalized communication from participant to the care team. Screen-positive women were offered treatment with low-dose aspirin (LDA; $81 \mathrm{mg}$ daily) and weekly intramuscular 17-hydroxyprogesterone (17-OHP; 250 mg each week) or nightly vaginal progesterone (200 mg each night) until 36 weeks. Compliance was encouraged by frequent communication and assessed by self-report. Women were similarly assessed at the second preterm birth prevention visit, and underwent ultrasound measurement of cervical length. If the cervical length was found to be less than $2.5 \mathrm{~cm}$, the women were evaluated for evidence of labor, considered for treatment with antenatal steroids, and offered vaginal progesterone if not on vaginal progesterone.

The estimated date of delivery was determined in accordance with American College of Obstetricians and Gynecologist guidelines. ${ }^{18}$ An adjudication committee consisting of three independent Maternal-Fetal Medicine physicians reviewed each PTB for final classification as spontaneous versus medically indicated.

The adaptive study design targeted a sample size of between 3,000 and 10,000 women to detect a reduction in sPTB from 6.4 to $4.7 \%$. The trial originally included interim assessments to select the final sample size. ${ }^{19}$ Depending on the variability observed, the adaptive design would stop at a sample size once futility or efficacy was determined. Due to insufficient funds, the study was terminated after 10 months of enrollment and prior to any unblinding and interim assessment of the data. The study analyses were focused on prespecified nonprimary outcomes of major clinical importance.

\section{Trial Oversight}

The investigators designed the trial and supervised its conduct, and they were wholly responsible for the interpretation of results. An independent Data Coordinating Center at the University of Utah aided in designing the study statistical plan and received limited-use data for analysis. An independent Data Safety and Monitoring Board comprised of two Maternal-Fetal Medicine physicians and a biostatistician not otherwise associated with the study met at prespecified time points to ensure the integrity and safety of the study. Sera Prognostics, Inc. funded the study, performed the serum proteomics screening tests, and reported results to study personnel. Sera personnel were not involved in any aspect of clinical care, participant data collection, or the 
determination of the pregnancy outcomes. Confidentiality agreements were in place between the authors and the sponsor. The trial was approved by the Intermountain Healthcare's institutional review board (1050481) and registered on ClinTrials.gov (NCT 03530332).

\section{PreTRM Test (Sera Prognostics, Inc.) and Risk Assignment}

Screening proteomics test results indicating a risk of SPTB of $\geq 14 \%$ were considered at increased risk for sPTB (screen positive). This cut-off was derived from a pilot study of the distribution of risk scores within the Utah population to be studied; it was determined that a risk cut-off of $\geq 14 \%$ would identify 20 to $30 \%$ of our Utah population as being at increased risk for SPTB.

\section{Patients}

Women with singleton pregnancy were eligible for participation if they were $>18$ years of age, had a cervical length $>2.5 \mathrm{~cm}$ at the time of mid-trimester ultrasound, had no medical contraindications to continuing pregnancy, and had intact membranes with no signs and/or symptoms of preterm labor (-Table 1 ).

Exclusion criteria included prior sPTB $<37$ weeks, cervical insufficiency, prior cervical conization, prior classical cesarean, known uterine anomaly, allergy to aspirin or 17-OHP, or a medical or obstetrical condition that the site investigator considered a contraindication due to an increased risk of PTB ( - Supplementary Table S1 [available in the online version]). Women also were ineligible if any of the following applied: progesterone use beyond $13^{6 / 7}$ weeks, use of a heparin agent, planned cesarean section or induction of labor prior to 37 weeks, planned cerclage placement, or a fetus with known major structural or genetic anomalies. Informed consent was obtained on all patients in the trial.

\section{Outcomes}

The primary outcome of the study was the proportion of participants experiencing sPTB $<37$ weeks due to preterm labor or preterm premature rupture of membranes in the absence of clinically evident infection, placental abruption, or other indications for preterm delivery.

The original secondary outcomes were the proportion of any PTB, total length of neonatal hospital stay for SPTB, and any PTB, respectively. These were changed on May 16, 2019 after discussion with investigators, lead statisticians, and representatives of Sera Prognostics, Inc. to GA at delivery, total length of neonatal stay, and neonatal intensive care unit (NICU) length of stay (LOS) among all neonates admitted to the NICU. These changes were instigated after reviewing results of independent studies indicating that in similar populations, the rate of SPTB was much lower than anticipated when we designed our study, making the possibility our study would have sufficient power to detect the primary outcome unlikely. All changes were made prior to reviewing unblinded data and appropriate documents (e.g., protocol and statistical analysis plan) were updated.
Exploratory outcomes comprised a variety of maternal and neonatal outcomes including neonatal LOS among PTBs or sPTBs, and the components of a published composite morbidity index that incorporates both complications of prematurity and NICU LOS. ${ }^{16}$

\section{Statistical Analysis}

The primary outcome was compared between study arms by using a Chi-squared test with an $\alpha$ threshold of 0.05 . During enrollment, no efficacy or futility assessments were performed per the original study design so the Type I error is maintained at 0.05 . The secondary and continuous exploratory outcomes are compared by using a Wilcoxon's ranksum test. Categorical exploratory outcomes were compared by using a Chi-squared test. When expected counts were low, Fisher's exact test was used. Lengths of stay were truncated to 28 days after the 40th week of gestation for neonates hospitalized longer than 28 days beyond the 40th week of gestation. Cases of stillbirth and neonatal death were given the worst possible score in the rank-based comparisons of LOS. Exploratory Kaplan-Meier estimates of NICU LOS were compared between study arms with the use of a log-rank test. Risk ratios and asymptotic 95\% confidence intervals (CIs) were calculated in SAS for the primary efficacy outcome. For related exploratory outcomes involving SPTB and any PTB rates, risk ratios and Melded 95\% CIs corresponding to Fisher's exact tests were calculated by using the exact $2 \times 2$ package in $\mathrm{R}^{20-22}$ All other analyses were performed by using SAS software, version 9.4 (SAS Institute). All $p$-values and $\mathrm{CIs}$ reported are two-sided. In that the trial was terminated, there were no corrections for multiple comparisons; the findings are meant to serve as hypothesis generating and are to be interpreted with caution.

\section{Results}

We approached 1,261 of 1,322 eligible women, consented 1,208 , and ultimately randomized 1,191 to undergo midtrimester serum proteomics screening $(n=595)$ or not ( $n=596$; - Fig. 1). Ten randomized women (six screened and four unscreened) delivered outside of our health care system, and outcome data were not available, leaving 1,181 pregnancies (99\%) available for analysis. Four women were later found to have a history of indicated, nonspontaneous PTB after review of records (one screened and three unscreened); these women remained in the study. As shown in - Supplementary Table S1 (available in the online version), screened and unscreened women were similar in demographically pertinent variables, including race, marital status, insurance type, education level, and approximate household income. Of note, $91.7 \%$ of participants were white (Hispanic and non-Hispanic) and less than 1\% Black or African-American. Over $80 \%$ were married, $79 \%$ had commercial insurance, and over $45 \%$ had a 4 -year college degree.

Of women who underwent serum proteomics screening, 198 (33.3\%) were screen positive and all were offered the sPTB risk-reduction protocol; 65.7\% attended the first and $64.1 \%$ attended the second prematurity prevention clinic 


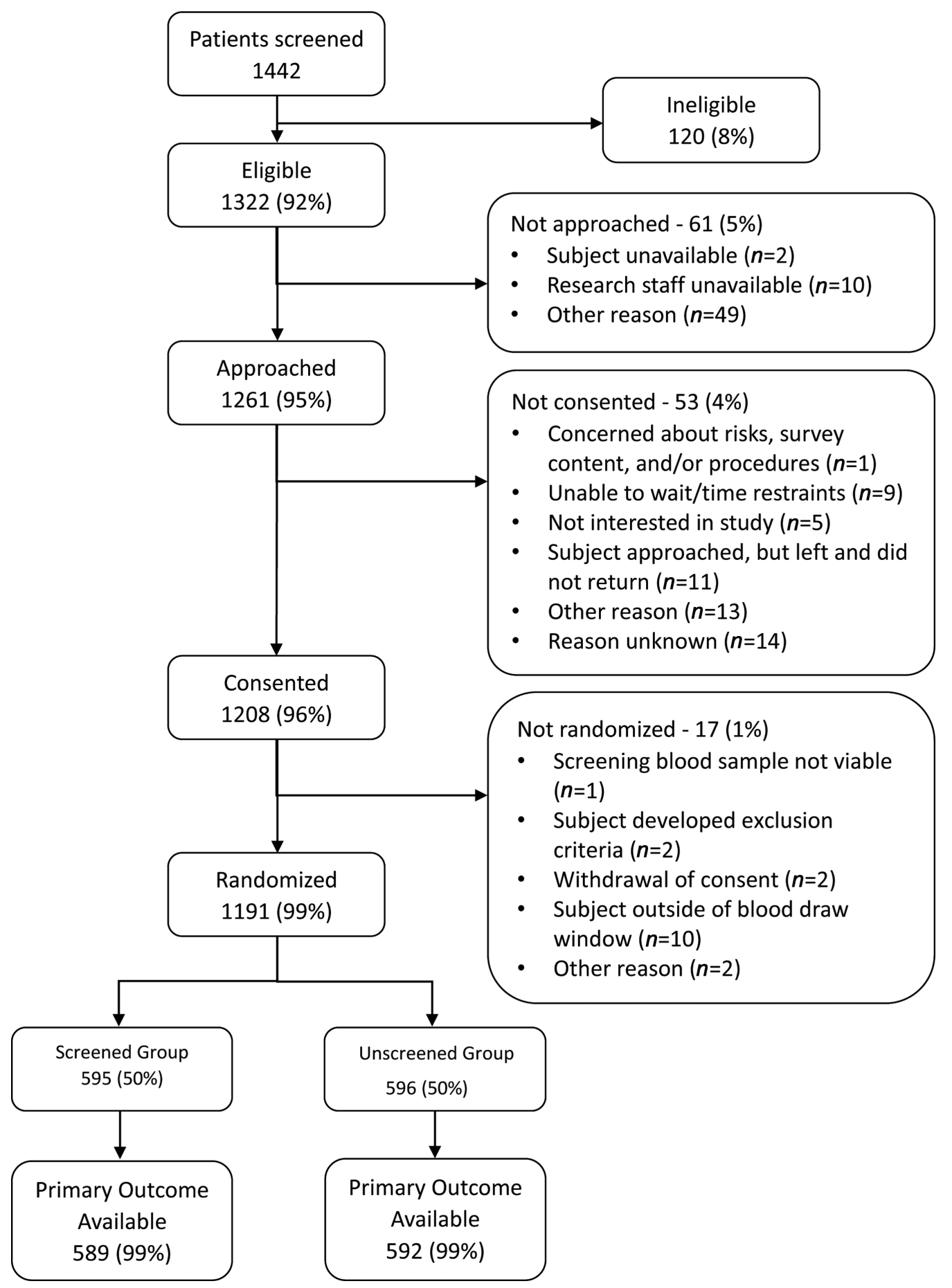

Fig. 1 Diagram of enrollment and randomization.

visit. In total, 39.4\% consented to treatment with 17-OHP, with $82.1 \%$ of these acknowledging no missed treatments; $66.7 \%$ consented to treatment with LDA, and $65.2 \%$ of these acknowledged no missed treatments. In total, 141 women (71\%) agreed to weekly contact.

The overall rate of SPTB $<37$ weeks in our population was 3.1\% (37 of 1,181 deliveries; - Fig. 2). sPTB occurred in 16 $(2.7 \%)$ of screened and 21 (3.5\%) of unscreened women $(p=0.413)$. The relative risk for sPTB was 0.77 (95\% CI: $0.40-1.45)$ for the screened versus the unscreened women. The risk difference was -0.008 ( -0.028 to 0.012 ). Therefore, while an $0.8 \%$ of absolute benefit was observed per the $95 \%$ $\mathrm{CI}$, we cannot rule out a benefit as high as $2.8 \%$ or as harmful as $1.2 \%$. Of the 196 screen-positive women, only four $(2.0 \%)$ had SPTB, and none were before 35 weeks. There were only five sPTBs $<34$ weeks in the entire study population, one in the screened group, and four in the unscreened group. No differences were found with regard to compliance with the PTB risk-reduction protocol. In total, 27 (4.6\%) and 36 (6.1\%) of screened and unscreened women, respectively, had PTB $<37$ weeks gestation for any reason (i.e., sPTB or PTB for other indications) ( $p=0.300)$, and PTB $<34$ weeks occurred in only 


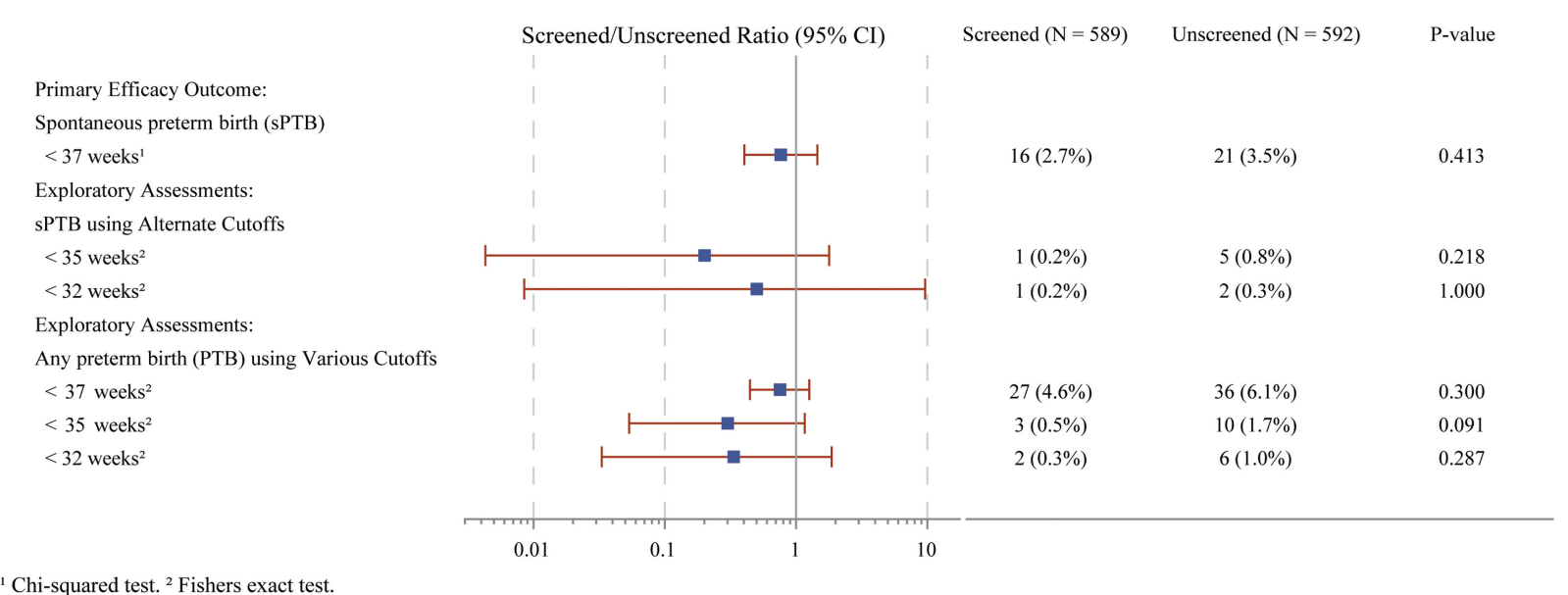

Fig. 2 Primary study efficacy outcome and additional exploratory assessments of treatment effect in screened and unscreened groups.

11 women (three in the screened group and eight in the unscreened group). There were no significant differences in indicated PTB between the screened and unscreened groups.

There were no significant differences between the screened and unscreened groups in the median GA at delivery $(p=0.464)$, the median NICU LOS among all neonates admitted to the NICU ( $p=0.231)$, or the median total length of neonatal stay for all infants admitted for any indication $(p=0.250 ;$-Table 2). Also, the median GA at delivery following any PTB or SPTB did not differ between the screened and unscreened groups ( $p=0.856$ and $p=0.951$, respectively). Using the prespecified Wilcoxon's rank-sum test, among the subgroup of infants admitted to the NICU after $\mathrm{SPTB}$, the median NICU LOS was significantly lower in

\begin{tabular}{|c|c|c|c|}
\hline & \multicolumn{3}{|l|}{ Group } \\
\hline & $\begin{array}{l}\text { Screened } \\
(n=589)\end{array}$ & $\begin{array}{l}\text { Unscreened } \\
(n=592)\end{array}$ & $p$-Value (comparing arms) \\
\hline \multicolumn{4}{|c|}{ Gestational age at delivery, median (IQR) } \\
\hline Any indication & $39.1(38.6-39.7)$ & $39.1(38.7-39.7)$ & $0.464 c^{\mathrm{a}}$ \\
\hline Following PTB & $36.4(35.1-36.6)$ & $36.1(34.3-36.7)$ & $0.856^{\mathrm{a}}$ \\
\hline Following sPTB & $36.4(35.5-36.6)$ & $36.1(35.1-36.7)$ & $0.951^{\mathrm{a}}$ \\
\hline \multicolumn{4}{|c|}{ NICU length of stay (d) ${ }^{b}$ among neonates admitted to the NICU: $n$, median (IQR) } \\
\hline Any indication & $\begin{array}{l}53 \\
6.0(3.7-8.0)\end{array}$ & $\begin{array}{l}45 \\
6.1(3.2-23.3)\end{array}$ & $0.231^{\mathrm{a}}$ \\
\hline Following PTB & $\begin{array}{l}10 \\
7.6(6.6-29.6)\end{array}$ & $\begin{array}{l}13 \\
36.7(17.2-52.0)\end{array}$ & $0.028^{\mathrm{a}}$ \\
\hline Following sPTB & $\begin{array}{l}6 \\
6.8(1.8-8.0)\end{array}$ & $\begin{array}{l}6 \\
45.5(34.6-79.0)\end{array}$ & $0.005^{\mathrm{a}}$ \\
\hline \multicolumn{4}{|c|}{ NICU length of stay $(\mathrm{d})^{\mathrm{b}}$ among all neonates, mean (SD) } \\
\hline Any indication & $0.7(3.83)$ & $1.4(9.48)$ & $0.494^{\mathrm{a}}$ \\
\hline Following PTB & $6.4(14.45)$ & $17.2(34.05)$ & $0.494^{\mathrm{a}}$ \\
\hline Following sPTB & $2.0(3.31)$ & $17.4(36.10)$ & $0.733^{\mathrm{a}}$ \\
\hline \multicolumn{4}{|c|}{ Total neonatal length of stay (d) ${ }^{b}$ among all neonates, median (IQR) } \\
\hline Any indication & $1.9(1.5-2.4)$ & $1.9(1.5-2.4)$ & $0.250^{\mathrm{a}}$ \\
\hline Following PTB & $2.2(1.9-7.2)$ & $3.4(2.0-35.6)$ & $0.087^{\mathrm{a}}$ \\
\hline Following sPTB & $2.2(1.9-6.9)$ & $3.1(2.1-17.2)$ & $0.257^{\mathrm{a}}$ \\
\hline
\end{tabular}

Abbreviations: SD, standard deviation; sPTB, spontaneous preterm birth.

aWilcoxon's rank sum test.

${ }^{\text {b} T r u n c a t e d ~ t o ~} 28$ days of life after the 40th week of gestation for those participants hospitalized longer than 28 days beyond the 40 th week of gestation. 
the screened compared with the unscreened group ( 6.8 days, IQR: $1.8-8.0$ vs. 45.5 days, IQR: $34.6-79.0 ; p=0.005$ ). Similarly, a significant median difference was observed among infants admitted after any PTB (7.6 days, IQR: 6.6-29.6 vs. 36.7 days, IQR: $17.2-52.0 ; p=0.028$ ). PTB infants in the screened group accumulated 172 total NICU days versus 619 total days in the unscreened group. Similarly, PTB infants in the screened versus unscreened groups spent 204 and 700 total days in the hospital before discharge, respectively.

Neonatal secondary outcomes among women undergoing screening are shown in -Table 3 . There were no significant differences between the screen positive and screen negative groups in median GA at delivery ( $p=0.241)$, the median NICU LOS among all neonates admitted to the $\operatorname{NICU}(p=0.359)$ or the median total length of neonatal stay for all infants $(p=0.432)$. The median GA at delivery following any PTB or SPTB did not differ between the screen positive and screen negative groups ( $p=0.455$ and $p=0.058$, respectively). There were no NICU admissions among screen positive women, whereas the median NICU LOS among the SPTB infants in the screen negative group was 6.8 days (IQR: $1.8-8.0$ ).

In an exploratory post hoc comparison of NICU LOS among all admitted neonates using the logrank test, which compares risks of events between arms over time and gives more weight to differences at the extreme tail of the NICU discharge distributions, the NICU LOS for all admitted neonates was significantly different between arms $(p=0.04)$ ( - Fig. 3A). In a similar post hoc analysis (-Fig. 3B, C), NICU LOS among all neonates admitted due to PTB or SPTB was significantly different between arms by log-rank test ( $p=0.03$ and $p<0.001$, respectively).

We also compared the severe neonatal composite morbidity and mortality index ${ }^{16}$ in the screened and unscreened groups. Among SPTB infants, a larger proportion of the screened group had lower, less morbid scores of 0 to 2 following SPTB compared with the unscreened group (93 vs. $76 \%$, respectively; - Table 4), and a smaller proportion of the screened group had a higher, more morbid score 3 or more ( 7 vs. $24 \%$, respectively), though the differences were not significant. Overall, the screened group had fewer scores of 3 or more (1\%) compared with the unscreened group (2\%), though again the difference was not significant $(p=0.061)$. The reduction in severe scores appeared to be associated with fewer infants having a NICU LOS more than 20 days in the screened compared with the unscreened group. There were no differences between the screened and unscreened groups with respect to other exploratory obstetric and neonatal outcomes.

Table 3 Neonatal secondary outcomes in screen-positive and screen-negative women

\section{Screened group}

Screen-positive

$(n=196)$

Gestational age at delivery, median (IQR)

$\begin{array}{ll}\text { Any indication } & 39.1(38.6-39.7) \\ \text { Following PTB } & 36.4(35.1-36.7) \\ \text { Following SPTB } & 36.6(36.5-36.7)\end{array}$

Screen-negative

$(n=393)$

$$
\begin{aligned}
& 39.1(38.6-39.7) \\
& 36.1(35.3-36.4)
\end{aligned}
$$$$
35.9(35.2-36.4)
$$

p-Value

$0.241^{\mathrm{a}}$

$0.455^{\mathrm{a}}$

$0.058^{a}$

NICU length of stay (days) ${ }^{b}$ among neonates admitted to the NICU: $n$, median (IQR)

$\begin{array}{lll}\text { Any indication } & 14 & 39 \\ \text { Following PTB } & 3.9(3.4-6.0) & 6.6(3.7-8.2) \\ & 2 & 8 \\ \text { Following SPTB } & 51.7(48.2-55.2) & 7.2(4.2-8.1) \\ & & 6 \\ & & 6.8(1.8-8.0)\end{array}$

NICU length of stay (days) $)^{b}$ among all neonates, mean (SD)

$\begin{array}{llll}\text { Any indication } & 0.9(5.59) & 0.7(2.54) & 0.213^{\mathrm{a}} \\ \text { Following PTB } & 9.4(20.97) & 4.3(7.58) & 0.150^{\mathrm{a}} \\ \text { Following SPTB } & 0.0(0.00)^{\mathrm{c}} & 2.7(3.61) & 0.071^{\mathrm{a}}\end{array}$

Total neonatal length of stay (days) ${ }^{\mathrm{b}}$ among all neonates, median (IQR)

\begin{tabular}{llll} 
Any indication & $1.8(1.5-2.2)$ & $1.9(1.5-2.4)$ & $0.432^{\mathrm{a}}$ \\
Following PTB & $1.8(1.7-2.2)$ & $2.7(2.0-7.6)$ & $0.061^{\mathrm{a}}$ \\
Following SPTB & $1.8(1.6-1.9)$ & $2.7(2.1-7.6)$ & $0.006^{\mathrm{a}}$ \\
\hline
\end{tabular}

Abbreviations: NICU, neonatal intensive care unit; SPTB, spontaneous preterm birth.

aWilcoxon's rank sum test.

bTuncated to 28 days of life after the 40 th week of gestation for those participants hospitalized longer than 28 days beyond the 40 th week of gestation.

'There were no NICU admissions among participants following sPTB in the screen-positive group. All participants in this group had a value of 0 for NICU length of stay (days). 


\section{A. All NICU admissions}

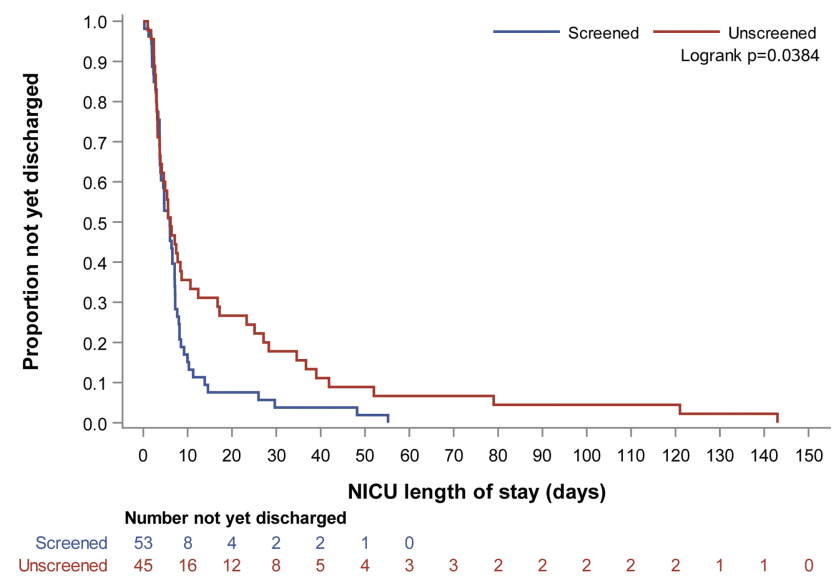

B. NICU admissions after PTB

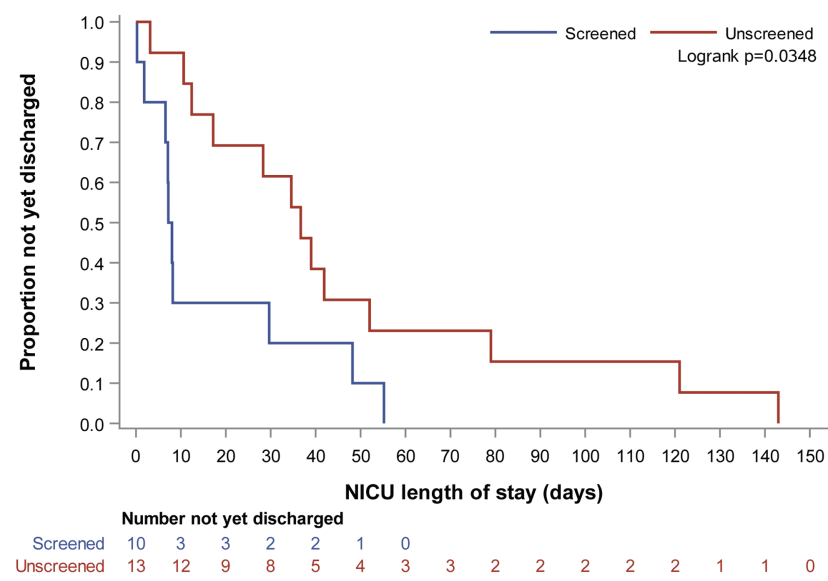

C. NICU admissions after SPTB

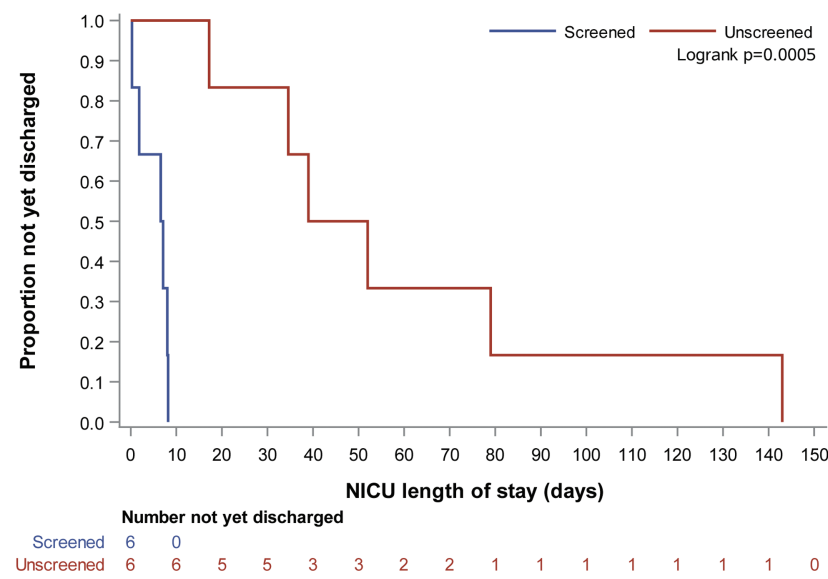

Fig. 3 KaplanMeier survival curves comparing length of neonatal intensive care unit stay (days) among screened (red line) and unscreened (blue line) groups. (A) Figure depicts outcomes for all births, (B) depicts outcomes following all PTBs for each group, and (C) depicts outcomes following SPTB in each group. sPTB, spontaneous preterm birth.

\section{Discussion}

\section{Principal Findings}

In our study of over 1,100 women with mid-trimester cervical length $\geq 2.5 \mathrm{~cm}$ and at otherwise low risk for $\mathrm{SPTB}$, the use of a validated serum proteomics screening test in the mid- trimester and a SPTB risk reduction protocol in screenpositive patients did not significantly reduce the rate of SPTB in screened women compared with women not screened. However, due to limited resources, the trial was stopped (fully blinded to outcomes across and between study arms) prior to achieving the prespecified target sample size for adequate statistical power to detect a difference if one exists. Despite this low sample size and the lower-thanprojected SPTB rate observed in our enrolled population, we noted that among the subgroup of infants admitted to the NICU after SPTB, the median LOS was significantly lower in the screened compared with the unscreened group. We posit that this is likely due to fewer sPTB infants $<35$ weeks at delivery in the screened compared with the unscreened patients, but the small number of cases does not allow other factors to be excluded. Though not a prespecified secondary outcome, this signal among the outcomes deserves further study.

\section{Results in Context of What is Known}

The increasing rate of PTB among pregnant women in the United States is at least partly due to the current tertiary approach to address this condition. A more effective strategy would be to risk-stratify women, early in the course of their pregnancy such that effective interventions could be initiated well before the onset of clinical signs and symptoms of SPTB. Other intervention trials have used various means of risk stratification using clinical risk scores, obstetric history, vaginal markers, vaginal infections, or the presence of a short cervix to identify patients at risk of preterm birth. Likewise, interventions to prevent SPTB in screen positive women, including nutritional supplements, patient education, antibiotics, weekly contact, cerclage, hormonal therapy, and uterine contraction monitoring have had inconsistent success. $^{5}$ However, the modest success of interventions like progesterone supplementation or cerclage to decrease the rate of recurrent SPTB among women with a history of a prior SPTB demonstrates the potential of such interventions in women at higher risk.

Ours is one of the first trials to assess the clinical utility of mid-trimester serum proteomics screening for risk of SPTB in women without acknowledged risk factors, and the first to offer screen-positive women plausible risk-reduction management. The serum proteomic screening test we used has been validated ${ }^{15}$ for the identification of women at higher risk for SPTB, but until now, there has been no evidence that such information can be used to safely improve neonatal outcomes. This is especially important given concerns regarding unnecessary or unproven care. ${ }^{23}$ Though we did not have power to assess whether serum screening reduced the rate of sPTB $<37$ weeks, our findings suggest shorter NICU LOS among admitted neonates from screen positive pregnancies. Indeed, primarily as a result of the shorter NICU LOS among those screened, we found that one of our exploratory outcomes, the composite neonatal morbidity index, was improved with regard to severe index scores. In turn, we speculate that this may be due to fewer PTBs prior to 35 weeks in the screened group. 


\begin{tabular}{|c|c|c|c|c|c|c|c|c|c|}
\hline & \multicolumn{3}{|l|}{ Overall } & \multicolumn{3}{|l|}{ PTB } & \multicolumn{3}{|l|}{ sPTB } \\
\hline & $\begin{array}{l}\text { Screened } \\
(n=584)\end{array}$ & $\begin{array}{l}\text { Unscreened } \\
(n=587)\end{array}$ & $p$-Value & $\begin{array}{l}\text { Screened } \\
(n=25)\end{array}$ & $\begin{array}{l}\text { Unscreened } \\
(n=36)\end{array}$ & $p$-Value & $\begin{array}{l}\text { Screened } \\
(n=15)\end{array}$ & $\begin{array}{l}\text { Unscreened } \\
(n=21)\end{array}$ & $p$-Value \\
\hline \multicolumn{10}{|l|}{$\begin{array}{l}\text { Composite } \\
\text { morbidity/ } \\
\text { mortality } \\
\text { score, n (\%) }\end{array}$} \\
\hline 0 & $511(88)$ & $517(88)$ & & $14(56)$ & $18(50)$ & & $8(53)$ & $13(62)$ & \\
\hline 1 & $44(8)$ & $41(7)$ & & $2(8)$ & $4(11)$ & & $2(13)$ & $2(10)$ & \\
\hline 2 & $24(4)$ & $15(3)$ & & $5(20)$ & $3(8)$ & & $4(27)$ & $1(5)$ & \\
\hline 3 & $4(1)$ & $11(2)$ & & $3(12)$ & $8(22)$ & & $0(0)$ & $4(19)$ & \\
\hline 4 & $1(0)$ & $3(1)$ & & $1(4)$ & $3(8)$ & & $1(7)$ & $1(5)$ & \\
\hline $\begin{array}{l}\text { Composite } \\
\text { morbidityl } \\
\text { mortality } \\
\text { score, } n(\%)\end{array}$ & & & $0.061^{a}$ & & & $0.238^{a}$ & & & $0.367^{a}$ \\
\hline $0-2$ & 579 (99) & $573(98)$ & & $21(84)$ & 25 (69) & & 14 (93) & $16(76)$ & \\
\hline $3-4$ & $5(1)$ & $14(2)$ & & $4(16)$ & $11(31)$ & & $1(7)$ & $5(24)$ & \\
\hline
\end{tabular}

Abbreviation: sPTB, spontaneous preterm birth.

aFisher's exact test.

\section{Strengths and Limitations}

The strengths of our study are several. We were able to consent and randomize over $90 \%$ of eligible women and to obtain outcome data on $99 \%$ of these. Our approach to riskreduction was straightforward and clinically pragmatic. Excluding the serum proteomics screening cost, maternal costs related to our risk-reduction management were quite modest. Over $90 \%$ of enrolled women delivered in a single health care system allowing confidence in pregnancy outcomes and costs. All preterm birth outcomes were independently adjudicated by Maternal-Fetal Medicine specialists.

This study has several limitations. First, it was prematurely terminated because of inadequate funding. Second, the rate of sPTB was just over 3\%, much less than the expected $6.4 \%$ rate we used in used in designing the study. Third, even though we observed a statistical difference in the NICU LOS among neonates admitted after SPTB, we did not adjust for multiple comparisons of all analyses performed, so results should be interpreted with caution. Finally, our findings may not be generalizable to the general U.S. population because our study population lacks ethnic diversity and over $75 \%$ of participants had commercial insurance.

\section{Conclusion}

In summary, we did not find that serum proteomic screening of a low risk population coupled with a SPTB risk-reduction protocol in screen positive patients resulted in a significantly lower rate of SPTB $<37$ weeks, though our study was underpowered. Our findings of a shortened NICU LOS following SPTB in the screened group are important exploratory outcomes that should prompt further study.
Funding

This study was funded by Sera Prognostics, Inc., Salt Lake City, UT.

Conflict of Interest

J.B. is employed by Sera Prognostics. G.L. was an employee of Sera Prognostics at the time of the study and holds stock in Sera Prognostics. S.E. holds stock in and serves on the Scientific Advisory Board for Sera Prognostics.

\section{References}

1 Prediction and prevention of preterm birth. Practice bulletin no. 130. American College of Obstetricians and Gynecologists. Obstet Gynecol 2012;120:964-973

2 Manuck TA, Rice MM, Bailit JL, et al; Eunice Kennedy Shriver National Institute of Child Health and Human Development Maternal-Fetal Medicine Units Network. Preterm neonatal morbidity and mortality by gestational age: a contemporary cohort. Am J Obstet Gynecol 2016;215(01):103.e1-103.e14

3 Boyle EM, Poulsen G, Field DJ, et al. Effects of gestational age at birth on health outcomes at 3 and 5 years of age: population based cohort study. BMJ 2012;344:e896-e896

4 Iams JD. Clinical practice. Prevention of preterm parturition. N Engl J Med 2014;370(03):254-261

5 Birth P. Causes, consequences, and prevention. In: Behrman RE, Bulter AS, eds. Institute of Medicine (US) Committee on Understanding Premature Birth and Assuring Healthy Outcomes. Washington, DC: National Academies Press; 2007

6 Ferrero DM, Larson J, Jacobsson B, et al. Cross-country individual participant analysis of 4.1 million singleton births in 5 countries with very high human development index confirms known associations but provides no biologic explanation for $2 / 3$ of all preterm births. PLoS One 2016;11(09):e0162506

7 Salihu H, Mbah AK, Alio AP, et al. Nulliparity and preterm birth in the era of obesity epidemic. J Matern Fetal Neonatal Med 2010;23 (12):1444-1450 
8 Esplin MS, Elovitz MA, Iams JD, et al; nuMoM2b Network. Predictive accuracy of serial transvaginal cervical lengths and quantitative vaginal fetal fibronectin levels for spontaneous preterm birth among nulliparous women. JAMA 2017;317(10): 1047-1056

9 Goldenberg RL, Culhane JF, Iams JD, Romero R. Epidemiology and causes of preterm birth. Lancet 2008;371(9606):75-84

10 Smith GCS, Shah I, White IR, Pell JP, Crossley JA, Dobbie R. Maternal and biochemical predictors of spontaneous preterm birth among nulliparous women: a systematic analysis in relation to the degree of prematurity. Int J Epidemiol 2006;35(05): 1169-1177

11 Dekker GA, Lee SY, North RA, McCowan LM, Simpson NAB, Roberts CT. Risk factors for preterm birth in an international prospective cohort of nulliparous women. PLoS One 2012;7(07): e39154

12 Bastek JA, Elovitz MA. The role and challenges of biomarkers in spontaneous preterm birth and preeclampsia. Fertil Steril 2013; 99(04):1117-1123

13 Mercer BM, Goldenberg RL, Das A, et al. The preterm prediction study: a clinical risk assessment system. Am J Obstet Gynecol 1996;174(06):1885-1893, discussion 1893-1895

14 Schaaf JM, Ravelli ACJ, Mol BWJ, Abu-Hanna A. Development of a prognostic model for predicting spontaneous singleton preterm birth. Eur J Obstet Gynecol Reprod Biol 2012;164(02):150-155

15 Saade GR, Boggess KA, Sullivan SA, et al. Development and validation of a spontaneous preterm delivery predictor in asymp- tomatic women. Am J Obstet Gynecol 2016;214(05):633.e1-633. e24

16 Hassan SS, Romero R, Vidyadhari D, et al; PREGNANT Trial. Vaginal progesterone reduces the rate of preterm birth in women with a sonographic short cervix: a multicenter, randomized, doubleblind, placebo-controlled trial. Ultrasound Obstet Gynecol 2011; 38(01):18-31

17 Markenson GR, Saade GR, Laurent LC, et al. Performance of a proteomic preterm delivery predictor in a large independent prospective cohort. Am J Obstet Gynecol MFM 2020;2(03):100140

18 American College of Obstetricians and Gynecologists. Committee opinion no 700: Methods for estimating the due date. Obstet Gynecol 2017;129(05):e150-e154

19 Broglio KR, Connor JT, Berry SM. Not too big, not too small: a goldilocks approach to sample size selection. J Biopharm Stat 2014;24(03):685-705

20 Fay MP, Proschan MA, Brittain E. Combining one-sample confidence procedures for inference in the two-sample case. Biometrics 2015;71(01):146-156

21 R Core Team. R: A language and environment for statistical computing. R Foundation for Statistical Computing, Vienna, Austria. Accessed 2013 at: http://www.R-project.org/

22 Chan ISF, Zhang Z. Test-based exact confidence intervals for the difference of two binomial proportions. Biometrics 1999;55(04): 1202-1209

23 Carroll AE. The high costs of unnecessary care. JAMA 2017;318 (18):1748-1749 